\title{
The potential toxic impact of different gadolinium-based contrast agents combined with 7-T MRI on isolated human lymphocytes
}

Björn Friebe ${ }^{1}$, Frank Godenschweger ${ }^{2}$, Mahsa Fatahi ${ }^{2}$, Oliver Speck ${ }^{2,3,4,5}$, Dirk Roggenbuck ${ }^{6,7}$, Dirk Reinhold ${ }^{8}$ and Annika Reddig ${ }^{8^{*}}$ (D)

\begin{abstract}
Background: To investigate a potentially amplifying genotoxic or cytotoxic effect of different gadolinium-based contrast agents (GBCAs) in combination with ultra-high-field 7-T magnetic resonance imaging (MRI) exposure in separated human peripheral blood lymphocytes.

Methods: This in vitro study was approved by the local ethics committee and written informed consent was obtained from all participants. Isolated lymphocytes from twelve healthy donors were incubated with gadobutrol, gadoterate meglumine, gadodiamide, gadopentetate dimeglumine, or gadoxetate either alone or combined with 7-T MRI (1 h). Deoxyribonucleic acid (DNA) double-strand breaks were assessed 15 min after MRI exposure by automated $\mathrm{YH} 2 \mathrm{AX}$ foci quantification. Cytotoxicity was determined at later endpoints by Annexin V/propidium iodide apoptosis assay $\left(24 \mathrm{~h}\right.$ ) and $\left[{ }^{3} \mathrm{H}\right]$-thymidine proliferation test $(72 \mathrm{~h})$. As a reference, lymphocytes from four different donors were exposed analogously to iodinated contrast agents (iomeprol, iopromide) in combination with computed tomography.

Results: Baseline $\mathrm{YH} 2 \mathrm{AX}$ levels ( $0.08 \pm 0.02 \mathrm{foci} / \mathrm{cell})$ were not significantly ( $p$ between 0.135 and 1.000 ) enhanced after administration of GBCAs regardless of MRI exposure. In contrast to the two investigated macrocyclic GBCAs, lymphocytes exposed to the three linear GBCAs showed a dose-dependent increase in apoptosis (maximum 186\% of unexposed control, $p<0.001$ ) and reduced proliferation rate (minimum $0.7 \%$ of unexposed control, $p<0.001$ ). However, additional 7-T MRI co-exposure did not alter GBCA-induced cytotoxicity.

Conclusions: Exposure of lymphocytes to different GBCAs did not reveal significant induction of $\mathrm{YH} 2 \mathrm{AX}$ foci, and enhanced cytotoxicity was only observed in lymphocytes treated with the linear GBCAs used in this study, independent of additional 7-T MRI co-exposure.
\end{abstract}

Keywords: Apoptosis, Contrast media, Lymphocytes, Magnetic resonance imaging, DNA damage

\section{Key points}

- No evidence of DNA damage after contrastenhanced 7-T MRI in vitro.

- Cytotoxicity was only observed after incubation with the three investigated linear GBCAs.

\footnotetext{
* Correspondence: annika.reddig@med.ovgu.de

${ }^{8}$ Institute of Molecular and Clinical Immunology, Otto von Guericke

University Magdeburg, Leipziger Str. 44, 39120 Magdeburg, Germany

Full list of author information is available at the end of the article
}

- Cytotoxicity was not further enhanced after combined exposure to 7-T MRI.

\section{Background}

As the number of magnetic resonance imaging (MRI) examinations and the applied field strength have been, and still are, increasing steadily, several questions about MRI safety as well as about the adverse effects of gadoliniumbased contrast agents (GBCAs) have raised public concerns $[1,2]$. Although the incidence of nephrogenic systemic fibrosis was strongly reduced by applying 
restrictive guidelines [3], new controversial data on the deposition of $\mathrm{Gd}$ in the brain and other tissues has accumulated [4-6].

Another major issue is whether electromagnetic fields applied in MRI can lead to genomic instability in humans. In recent years, several studies have investigated the genotoxic impact of MRI exposure on lymphocytes in vivo and in vitro, as reviewed in detail elsewhere [7-10]. While some authors reported enhanced Deoxyribonucleic acid (DNA) damage in human lymphocytes after MRI examination [11-14], others could not confirm these findings [15-19]. Until now, no clear evidence or mechanism about MRI-induced DNA lesions could be described. Furthermore, most studies did not compare unenhanced and contrast-enhanced MRI exposure. Reports investigating radiation damage after computed tomography (CT) demonstrated an amplification of DNA lesions in the presence of iodinated contrast agent (ICA), probably due to enhanced photoelectric absorption of $\mathrm{x}$-rays by injected iodine atoms [20-23]. But whether GBCAs also alter a potential toxic effect induced by MRI or vice versa needs to be investigated in greater detail. An in vivo study by Yildiz et al. [24] revealed no significant increase in DNA damage in patients after uenhanced MRI, but instead a significant induction of DNA lesions when MRI exposure was combined with injection of non-ionic, linear gadodiamide. In contrast, no alterations in DNA double-strand break (DSB) formation assessed by $\gamma \mathrm{H} 2 \mathrm{AX}$ immunofluorescence microscopy were found by an in vivo study by Reddig et al. [19], regardless of administration of the macrocyclic GBCA gadobutrol. Nevertheless, studies investigating the impact of different kinds of GBCAs on lymphocyte toxicity, as well as data about the genotoxic or cytotoxic impact of various GBCAs in combination with ultra-high-field MRI, are rare. We applied ultra-high-field MRI to the potential effects, as previous publications [11-19] have been contradictory regarding the induction of biological effects.

Therefore, the aim of this in vitro study was to investigate a potentially amplifying effect on $\gamma \mathrm{H} 2 \mathrm{AX}$ foci formation or cytotoxicity induced by different classes of GBCAs in combination with ultra-high-field MRI exposure at $7 \mathrm{~T}$ in human peripheral blood lymphocytes.

\section{Methods}

\section{Study design and participants}

This in vitro study was approved by the local Ethics Committee (RAD 244) and written informed consent was obtained from all participants. Heparinised blood samples were collected from twelve healthy subjects (six women, six men; mean age 35.8 years) on the day of examination. Each donor sample was subdivided and finally incubated with seven different agents (five GBCAs, Mannitol 780 and Mannitol 1960) in two different concentrations, plus a positive and a negative control (16 samples per donor). These samples were divided into one group that was exposed to 7-T MRI and another group that was not exposed to 7-T MRI, resulting in 32 different experiments for each of the twelve donors (see below).

\section{Lymphocyte isolation}

Peripheral blood mononuclear cells, here referred to as lymphocytes, were separated by density gradient centrifugation and suspended in standard RPMI-1640 medium supplemented with $10 \%$ fetal calf serum, $100 \mathrm{U} / \mathrm{mL}$ penicillin and $100 \mu \mathrm{g} / \mathrm{mL}$ streptomycin before MRI/CT scan, thereby enabling immediate assessment of DNA damage and lymphocyte activation after exposure.

\section{Contrast media and exposure conditions}

As different chemical structures of GBCA are considered to have different risk profiles regarding nephrogenic systemic fibrosis and $\mathrm{Gd}$ deposition [4-6], we evaluated all four structural classes of GBCA (linear/ macrocyclic; ionic/non-ionic). Five different GBCAs (Table 1) that were available in our department were utilised as ready-to-use solutions for injection as applied in clinics, in order to study a setting that is as close to a realistic clinical setting as possible. MRI contrast agents were applied at a physiological concentration of $2 \mathrm{mM}$, estimated assuming a recommended diagnostic dose in patients of $0.1 \mathrm{mmol}$ GBCA per kilogram body weight distributed in $60-80 \mathrm{~mL}$ blood per kilogram body weight [25], as well as a tenfold higher dosage of $20 \mathrm{mM}$. Mannitol solutions, adjusted to lowest (gadodiamide; mannitol-780 $\mathrm{mOsm} / \mathrm{kg} \mathrm{H} \mathrm{H}_{2} \mathrm{O}$ ) and highest (gadopentetate dimeglumine; mannitol$1960 \mathrm{mOsm} / \mathrm{kg} \mathrm{H} \mathrm{H}_{2} \mathrm{O}$ ) final osmolality of applied contrast agents, served as controls.

Lymphocytes and contrast agent were transferred into 96-well plates. Approximately $30 \mathrm{~min}$ after contrast agent administration one half of the samples was placed into the isocentre of a whole-body 7-T MRI scanner (Siemens Healthineers, Erlangen, Germany), whereas the other half was placed outside the magnet shielding at a distance of $8 \mathrm{~m}$ from the isocentre in a low magnetic field of approximately $50 \mathrm{mT}$, but within the scanning room to provide identical temperature conditions.

MRI exposure was performed in a 1 -h scan procedure. To increase potential toxic effects, an echo planar imaging sequence was adjusted to the maximum permissible switched gradient and specific absorption rate, as described previously by Reddig et al. [17]. Twelve experiments with lymphocytes from different donors were performed. 
Table 1 List of contrast agents applied in this in vitro study

\begin{tabular}{|c|c|c|c|c|c|c|c|}
\hline Imaging system & Chemical name & $\begin{array}{l}\text { Trade name } \\
\text { (manufacturer) }\end{array}$ & Structure, ionicity & Initial concentration & $\begin{array}{l}\text { Osmolality (mOsm/ } \\
\mathrm{kg} \mathrm{H}_{2} \mathrm{O} \text { ) }\end{array}$ & $\begin{array}{l}\text { Viscosity (mPa.s, } \\
\left.37^{\circ} \mathrm{C}\right)\end{array}$ & Clearance \\
\hline MRI & Gadobutrol & $\begin{array}{l}\text { Gadovist (Bayer Vital, } \\
\text { Leverkusen, Germany) }\end{array}$ & Macrocyclic, nonionic & $1.0 \mathrm{mmol} / \mathrm{mL}$ & 1603 & 5.0 & Renal \\
\hline MRI & $\begin{array}{l}\text { Gadoterate } \\
\text { meglumine }\end{array}$ & $\begin{array}{l}\text { Dotarem (Guerbet, } \\
\text { Roissy, France) }\end{array}$ & Macrocyclic, ionic & $0.5 \mathrm{mmol} / \mathrm{mL}$ & 1350 & 2.0 & Renal \\
\hline MRI & Gadodiamide & $\begin{array}{l}\text { Omniscan (General } \\
\text { Electric Healthcare Buchler, } \\
\text { Braunschweig Germany) }\end{array}$ & Linear, nonionic & $0.5 \mathrm{mmol} / \mathrm{mL}$ & 780 & 1.4 & Renal \\
\hline MRI & $\begin{array}{l}\text { Gadopentetate } \\
\text { dimeglumine }\end{array}$ & $\begin{array}{l}\text { Magnograf (Jenapharm, } \\
\text { Jena, Germany) }\end{array}$ & Linear, ionic & $0.5 \mathrm{mmol} / \mathrm{mL}$ & 1960 & 2.9 & Renal \\
\hline MRI & Gadoxetate & $\begin{array}{l}\text { Primovist (Bayer Pharma, } \\
\text { Berlin, Germany) }\end{array}$ & Linear, ionic & $0.25 \mathrm{mmol} / \mathrm{mL}$ & 688 & 1.2 & $\begin{array}{l}50 \% \text { hepatic, } \\
50 \% \text { renal }\end{array}$ \\
\hline CT & lomeprol & $\begin{array}{l}\text { Imeron } 300 \mathrm{M} \text { (Bracco } \\
\text { Imaging, Konstanz, Germany) }\end{array}$ & Monomeric, nonionic & $300 \mathrm{mg}$ iodine/mL & 521 & 2.9 & Renal \\
\hline CT & lopromide & $\begin{array}{l}\text { Ultravist } 300 \text { (Bayer Vital, } \\
\text { Leverkusen, Germany) }\end{array}$ & Monomeric, nonionic & $300 \mathrm{mg}$ iodine/mL & 590 & 4.7 & Renal \\
\hline
\end{tabular}

MRI magnetic resonance imaging, CT computed tomography

As a control, analogously to MRI experiments, lymphocytes from four different donors were irradiated with $\mathrm{x}$-rays by conducting a spiral CT scan (Aquilion Prime, Toshiba Medical Systems, Tustin, CA, USA). Here, two different ICAs were investigated (Table 1) at final doses of 5,15 , and $50 \mathrm{mg}$ iodine per $\mathrm{mL}$ referring to previous studies $[20,23,26]$. Mannitol controls with the same osmolality as iopromid $\left(590 \mathrm{mOsm} / \mathrm{kg} \mathrm{H}_{2} \mathrm{O}\right.$ ) were included. To reach a high dose concentration of $50 \mathrm{mg}$ iodine per mililitre with conventional contrast agent $(300 \mathrm{mg} \mathrm{I} / \mathrm{mL})$, a large volume fraction of contrast agent solution needed to be added, representing one-sixth of the cell suspension.

After MRI/CT exposure, samples were transported to the laboratory within $10 \mathrm{~min}$ in a styrofoam box and processed immediately. Additionally, lymphocytes exposed prior to the MRI/CT scan with a DSB-inducing dose of $0.5 \mathrm{~Gy} \gamma$-radiation or the apoptosis-inducing cytostatic drug camptothecin $(\mathrm{CPT}, 2 \mu \mathrm{M})$ were used as positive controls for the genotoxic and cytotoxicity assays, respectively.

\section{$\mathrm{H} 2 \mathrm{AX}$ immunofluorescence analysis}

For genotoxicity assessment, $\gamma \mathrm{H} 2 \mathrm{AX}$ foci were quantified as a marker for DNA DSBs. Samples irradiated with 0.5 Gy $\gamma$-radiation served as positive controls. To allow phosphorylation after MRI/CT exposure, cell cultures were incubated in plates with 96 wells for an additional 15 min at $37{ }^{\circ} \mathrm{C}$ in $7 \% \mathrm{CO}_{2}$. Afterwards, samples were washed in phosphate-buffered saline (PBS) and pipetted onto microscope slides. After fixation in $2 \%$ formaldehyde $(15 \mathrm{~min})$ and permeabilisation in $0.2 \%$ Triton $\mathrm{X}-100$, cells were blocked in PBS containing 1\% bovine serum albumin. Samples were incubated with an anti-phosphohistone H2AX mouse monoclonal IgG primary antibody (Millipore, Schwalbach, Germany; dilution 1:500) overnight at $4{ }^{\circ} \mathrm{C}$. After washing in blocking buffer, slides were stained with a polyclonal goat anti-mouse IgG antibody conjugated to Alexa Fluor 488 (Lifetechnologies, Darmstadt, Germany; dilution 1:500) for $1 \mathrm{~h}$ at room temperature. Subsequently, slides were washed in PBS and covered with 4'6-diamidino-2-phenylindole-containing mounting medium. The amount of $\gamma \mathrm{H} 2 \mathrm{AX}$ foci was quantified from an average of 370 lymphocytes per sample by an automated digital microscopy system, based on z-stack images of five focal planes throughout each nucleus (AKLIDES; Medipan, Dahlewitz, Germany) [27].

\section{Apoptosis detection}

An Annexin V/propidium iodide (PI) staining kit (Biolegend, San Diego, CA, USA) was used to assess cell viability $24 \mathrm{~h}$ after exposure. Samples simultaneously incubated with $2 \mu \mathrm{M}$ CPT were used as positive controls. Lymphocytes were stained according to the manufacturer's instructions and analysed by flow cytometry (BD LSRFortessa; BD Biosciences, Mountain View, CA, USA). A minimum of 15,000 lymphocytes per sample were classified. Since the rate of necrotic cells $\left(\mathrm{PI}^{+}\right.$, Annexin $\mathrm{V}^{-}$) was below $1 \%$, only the sum of early ( $\mathrm{PI}^{-}$, Annexin $\left.\mathrm{V}^{+}\right)$and late $\left(\mathrm{PI}^{+}\right.$, Annexin $\left.\mathrm{V}^{+}\right)$apoptotic cells was used for evaluation.

\section{Proliferation assay}

A standard tritiated thymidine $\left({ }^{3}[\mathrm{H}]-\mathrm{TdR}\right)$ incorporation assay was used to analyse the level of DNA synthesis. In brief, lymphocytes had to be stimulated with $2 \mu \mathrm{g} / \mathrm{mL}$ phytohemagglutinin (PHA) directly after MRI/CT exposure. After 3 days the cells were pulsed with $\left[{ }^{3} \mathrm{H}\right]-\mathrm{TdR}(0.2 \mu \mathrm{Ci} /$ well $)$ for $6 \mathrm{~h}$ and subsequently harvested. For quantification the microplate liquid scintillation counter Wallac MicroBeta TriLux from Perkin Elmer was utilised (Waltham, MA, USA). 
Lymphocytes incubated with $2 \mu \mathrm{M}$ CPT served as positive controls.

\section{Statistical analysis}

Statistical analysis was performed by Graph Pad Prism software version 5.01 (Graph Pad Software, La Jolla, CA, USA) and all data in text and figures are presented as mean \pm standard error of the mean. For comparison of negative control samples with multiple treatment conditions, significance levels were calculated by repeated measures ANOVA with a confidence interval of $95 \%$, followed by Dunnett's post hoc test. When single exposure conditions induced significant differences, direct comparison of matching samples with and without CT/MRI exposure was performed, using paired $t$-tests to detect potential additional or synergistic effects.

\section{Results}

Toxic impact of iodinated contrast agent during CT

To compare our data obtained from ultra-high-field MRI with exposure to ionising radiation and the published literature, we exposed lymphocytes to $\mathrm{CT}$ in the presence or absence of ICA at doses of 5, 15 and $50 \mathrm{mg}$ iodine $/ \mathrm{mL}$ as a control. As expected, CT exposure alone significantly increased the number of $\gamma \mathrm{H} 2 \mathrm{AX}$ foci from an initial $0.04 \pm 0.01$ (mean \pm standard error of the mean) to $0.34 \pm 0.02$ foci per cell (Fig. 1a; Additional file 1: Table S1). The presence of ICA further enhanced $\gamma \mathrm{H} 2 \mathrm{AX}$ foci formation compared to CT exposure alone, with the strongest effect at the nonphysiological high dose of $50 \mathrm{mg} \mathrm{I} / \mathrm{mL}$ (iomeprol-50 + CT, $1.28 \pm 0.06$ foci/cell; iopromide- $50+\mathrm{CT}, 1.23 \pm 0.02$ foci/cell; $p<0.001)$. In contrast, mannitol treatment or ICA without additional radiation did not alter baseline $\gamma \mathrm{H} 2 \mathrm{AX}$ levels significantly.

Determination of apoptotic cells $24 \mathrm{~h}$ after exposure revealed no significant differences in level of Annexin $\mathrm{V}^{+}$cells between untreated $(9.5 \pm 1.6 \%)$ and $\mathrm{CT}$ exposed samples $(8.5 \pm 2.0 \%)$ (Fig. 1b; Additional file 1: Table S2). However, incubation of lymphocytes with ICA alone led to a dose-dependent induction of apoptosis, which was further increased for very high dose ICA (50 $\mathrm{mg} \mathrm{I} / \mathrm{mL}$ ) in combination with CT exposure (iomeprol-50, $21.4 \pm 1.9 \%$ vs iomeprol-50 + CT, $24.3 \pm 2.3 \%, p=$ 0.008 ; iopromide $-50,16.9 \pm 2.6 \%$ vs iopromide $-50+\mathrm{CT}$, $20.2 \pm 3.2 \%, p=0.099)$. Here, apoptosis rate was comparable to CPT-treated cells (CPT, $19.7 \pm 2.6 \%$ vs CPT + CT, $20.8 \pm 2.7 \%, p=0.031$ ).

A significant reduction in DNA synthesis was only observed at the highest applied dose of ICA (iomeprol-50, $48 \pm 7 \%$; iomeprol- $50+\mathrm{CT}, 43 \pm 6 \%$; iopromide$50, \quad 27 \pm 3 \% ; \quad$ iopromide $-50+\mathrm{CT}, \quad 26 \pm 3 \% ; \quad p<0.001$; Fig. 1c; Additional file 1: Table S3). As shown by paired $t$-tests, this effect was further significantly enhanced for iomeprol when lymphocytes were additionally irradiated by CT $(p=0.022)$. In contrast to the apoptosis assessment of unstimulated cells, CPT treatment affected proliferating cells much more strongly compared to high dose ICA, resulting in proliferation rates below the quantification limit $(<2 \%)$, and a high dose of mannitol inhibited lymphocyte proliferation (mannitol-50, 84 \pm $7 \%, p=0.016$; mannitol-50 + CT, $85 \pm 7 \%, p=0.020$ ), an effect which was probably due to the high amount of mannitol and volume fraction of one-sixth of the whole cell suspension (Fig. 1c; Additional file 1: Table S3).

\section{Toxic impact of gadolinium-based contrast agent during 7-T MRI}

To investigate the impact of GBCAs on isolated lymphocytes, samples were incubated with different classes of GBCAs at a dose of $2 \mathrm{mM}$ and $20 \mathrm{mM}$ for $1 \mathrm{~h}$ with and without combined 7-T MRI exposure. Data revealed no significant induction of $\gamma \mathrm{H} 2 \mathrm{AX}$ foci (negative control, $0.08 \pm 0.02$ foci/cell) in any sample, independently of the GBCA structure, GBCA concentration or 7-T MRI exposure, whereas the number of DSBs was enhanced after $\gamma$-radiation (Fig. 2a; Additional file 1: Table S4). Surprisingly, lymphocytes treated with $2 \mathrm{mM}$ or $20 \mathrm{mM}$ gadodiamide showed a slight but significant reduction in $\gamma \mathrm{H} 2 \mathrm{AX}$ levels (gadodiamide- 2 and $-20,0.04 \pm 0.01 \mathrm{foci} /$ cell, $p=0.030$ and $p=0.012$, respectively).

Assessment of apoptosis rate indicated reduced cell viability $(p<0.01)$ when lymphocytes were incubated with $2 \mathrm{mM}$ gadodiamide as well as after treatment with $20 \mathrm{mM}$ of the three linear GBCAs applied in this study (Fig. 2b; Additional file 1: Table S5). However, additional 7-T MRI exposure did not alter apoptosis rates compared to GBCA or CPT treatment alone. Statistical analysis even indicated a slight decrease in apoptosis when lymphocytes were cultured in the presence of the macrocyclic gadobutrol.

Compared to unexposed controls (100\%), analysis of proliferation rates after $72 \mathrm{~h}$ of lymphocytes exposed to the three investigated linear GBCAs showed a strong, dose-dependent decrease in cell division, reaching levels below the quantification limit at concentrations of $20 \mathrm{mM}$, similar to positive CPT control (Fig. 2c; Additional file 1: Table S6). Whereas ICA and linear GBCAs induced apoptosis of unstimulated lymphocytes to a similar extent, DNA synthesis of the PHA-activated lymphocytes was inhibited more strongly by the linear GBCAs (gadodiamide, gadopentetate dimeglumine, gadoxetate) compared to applied ICA. However, a small but significant stimulatory effect was observed in activated lymphocytes incubated for $72 \mathrm{~h}$ with $20 \mathrm{mM}$ of macrocyclic gadoterate. 

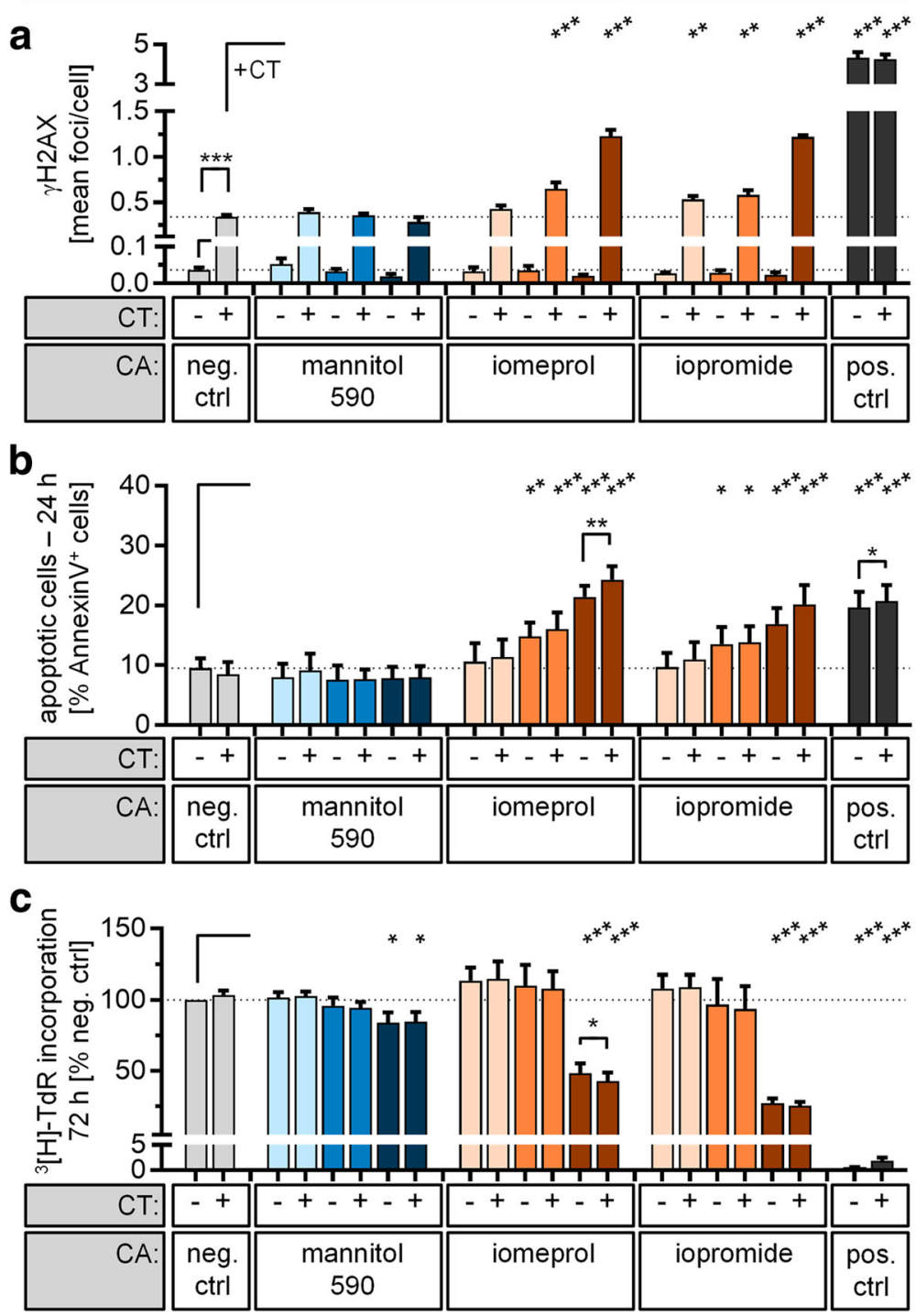

Fig. 1 Genotoxic and cytotoxic impact of contrast-enhanced CT exposure. Isolated lymphocytes were incubated with indicated concentrations of iomeprol or iopromid. Samples incubated in cell culture medium only (neg. ctrl) or with mannitol solution with the same osmolality as iopromide ( $590 \mathrm{mOsm} / \mathrm{kg} \mathrm{H}_{2} \mathrm{O}$ ) served as negative controls. Additionally, samples were either irradiated by a thorax CT scan (+) or placed outside the CT scanner (-) at the same temperature. a The level of DNA double-strand breaks was assessed 15 min after $\mathrm{CT}$ exposure by $\mathrm{\gamma H} 2 \mathrm{AX}$ staining and automated foci quantification. Lymphocytes irradiated with 0.5 Gy served as positive controls (pos. ctr). b Apoptosis rate was quantified after $24 \mathrm{~h}$ by Annexin V/propidium iodide staining combined with flow cytometric analysis. Samples incubated with $2 \mu \mathrm{M}$ camptothecin served as positive controls. c For proliferation analysis, lymphocytes were activated with PHA directly after CT exposure. Level of DNA synthesis was determined after $72 \mathrm{~h}$ by $\left[^{3} \mathrm{H}\right]$-thymidine incorporation. Lymphocytes treated with $2 \mu \mathrm{M}$ camptothecin served as positive controls. Diagrams displays mean \pm standard error of the mean of four experiments $\left({ }^{* * *} p \leq 0.001 ;{ }^{* *} p \leq 0.01 ;{ }^{*} p \leq 0.05\right)$ and raw data are listed in Additional file 1 : Tables S1-S3

\section{Discussion}

Although MRI is considered a safe, non-invasive diagnostic imaging technique, in contrast to approaches based on ionising radiation exposure, several concerns about potential MRI-related health risks have arisen in the past few years and have not yet been sufficiently clarified to date. As a potential genotoxic effect of MRI would have a large impact on the clinical practice of MRI, several studies have been conducted in the past decade. Whereas some studies did not find any significant increase in genetic damage after MRI exposure, other studies reported a significant genotoxic MRI-related effect [11-19, 24, 28]. These studies, however, show a large diversity regarding field strengths, exposure 


\section{mannitol control: $\square 2 \square 20 \quad$ gadolinium-based contrast agent [mM]: $\square 2 \square 20$}

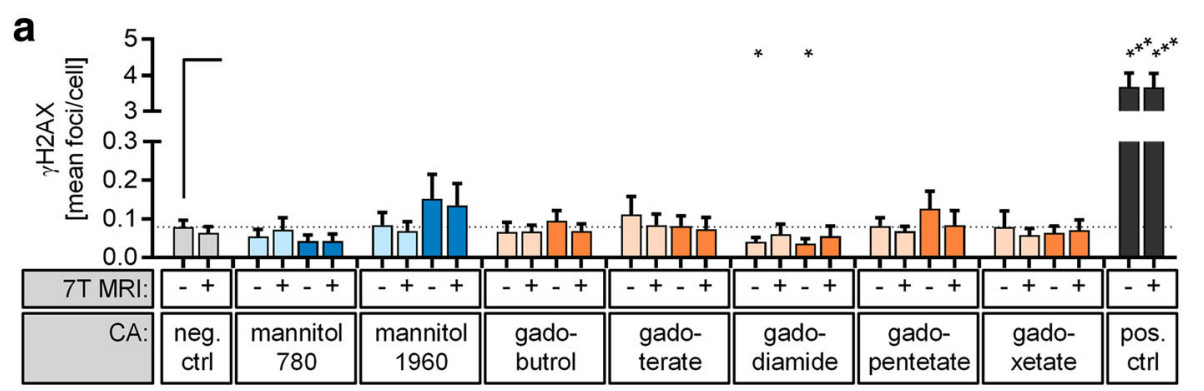

$\mathbf{b}_{\mathcal{L}}$

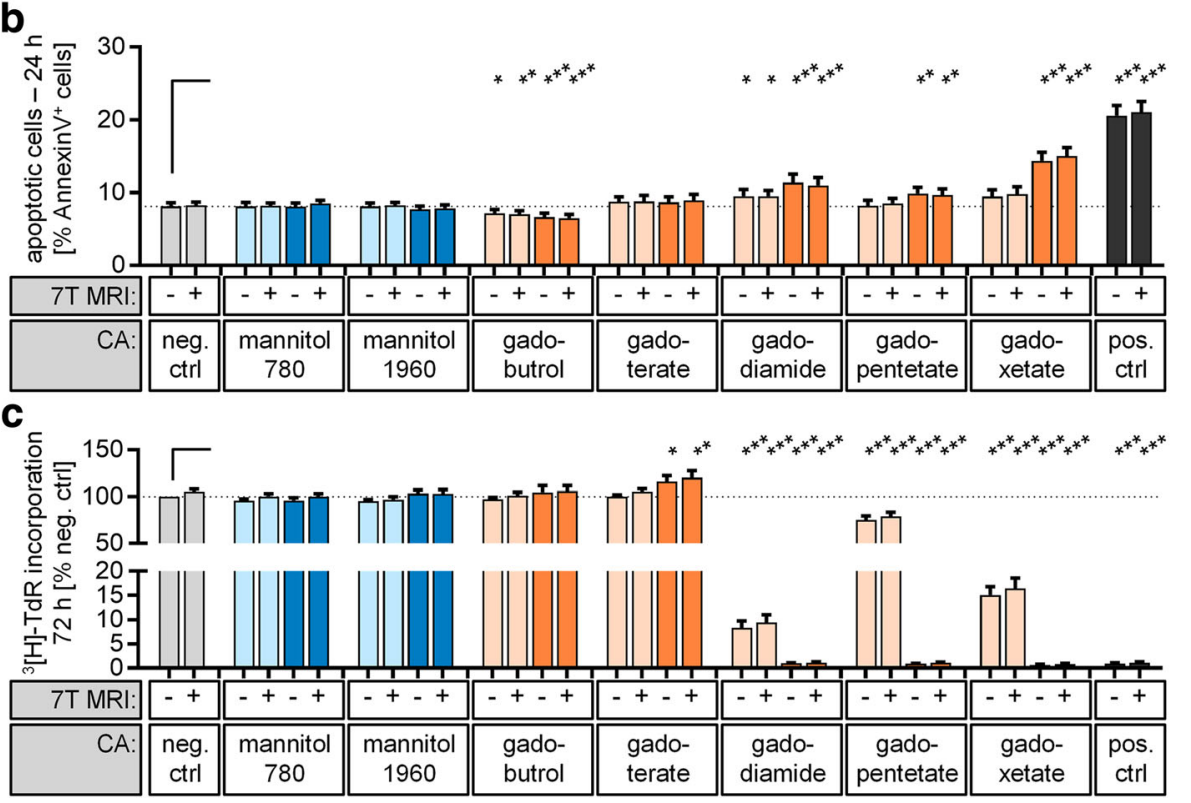

Fig. 2 Genotoxic and cytotoxic impact of contrast-enhanced 7-T MRI exposure. Isolated lymphocytes were incubated with the indicated class and concentration of gadolinium-based contrast agent. Samples incubated in cell culture medium only (neg. ctrl) or with mannitol solution comparable with the lowest ( $780 \mathrm{mOsm} / \mathrm{kg} \mathrm{H}_{2} \mathrm{O}$; gadodiamide) and highest ( $1960 \mathrm{mOsm} / \mathrm{kg} \mathrm{H}_{2} \mathrm{O}$; gadopentetate dimeglumine) osmolality of GBCAs served as controls. Additionally, samples were either exposed to 7-T MRI (+) or placed outside the MRI scanner (-) at the same temperature. a The level of DNA double-strand breaks was assessed $15 \mathrm{~min}$ after exposure by $\mathrm{\gamma H} 2 \mathrm{AX}$ staining and automated foci quantification. Lymphocytes irradiated with $0.5 \mathrm{~Gy}$ served as positive controls (pos. ctrl). b Apoptosis rate was quantified after $24 \mathrm{~h}$ by Annexin V/propidium iodide staining combined with flow cytometric analysis. Samples treated with $2 \mu \mathrm{M}$ camptothecin served as positive controls. c For proliferation analysis, lymphocytes were activated with PHA directly after MRI exposure. Level of DNA synthesis was determined after $72 \mathrm{~h}$ by $\left[{ }^{3} \mathrm{H}\right]$-thymidine incorporation. Lymphocytes treated with $2 \mu \mathrm{M}$ camptothecin served as positive controls. Diagrams display mean \pm standard error of the mean of 12 experiments $\left({ }^{* *} p \leq 0.001 ;{ }^{* *} p \leq 0.01 ;{ }^{*} p \leq 0.05\right)$ and raw data are listed in Additional file 1: Tables S4-S6

parameters, and types of genotoxicity analysis. In addition, no established hypothesis for a potential mechanism can explain the results of the studies where significant effects were found.

Furthermore, it is known that no single genotoxic endpoint (e.g., single/double-stand breaks, chromosomal aberrations, micronuclei) allows one to determine the carcinogenic risk of an agent. Consequently, further studies that try to investigate possible underlying mechanisms of genotoxicity should divide one sample into multiple aliquots in order to investigate several end-points of genotoxicity and cytotoxicity at the same time [7]. In this context, researchers also have to look for other possible genotoxic endpoints, such as oxidative stress, as has been reported by Erdamar et al. in 2014 [29].

As two studies that observed DNA damage were conducted after contrast-enhanced MRI [13, 24], a potential cause could also lie in a possible interaction mechanism with contrast media or in an interaction of contrast media with MRI. Therefore, in this study we examined the genotoxic and cytotoxic impact of different GBCAs on human blood lymphocytes and analysed a potential additive or synergistic effect when simultaneously exposed to 7-T MRI. 
At our institution, ultra-high-field MRI is applied for research purposes only and administration of contrast agents is prohibited in studies conducted in vivo on humans. Thus, the current investigations had to be performed under in vitro conditions. However, the use of isolated, unshielded lymphocytes, the constant presence of high GBCA concentrations and the application of 7-T MRI combined with the maximum permissible switched gradient and specific absorption rate all present enhanced exposure conditions in these samples compared to in vivo examinations, and would presumably also maximised the level of cell damage and detectability.

Genotoxicity was determined by $\gamma \mathrm{H} 2 \mathrm{AX}$ staining, which is one of the most sensitive biomarkers for DNA DSB assessment. Our experiments confirmed the induction of $\gamma \mathrm{H} 2 \mathrm{AX}$ foci after CT exposure and an additional dose-dependent increase in the presence of ICA, while ICA alone did not lead to $\gamma \mathrm{H} 2 \mathrm{AX}$ formation. As has also been shown in previous publications [20-22], the extent of DNA damage was dependent on irradiation dose as well as on ICA concentration. DSB induced by unenhanced CT were mainly repaired and did not alter apoptosis or proliferation rate, whereas ICA-induced cytotoxicity was partially enhanced for high dose ICA in combination with CT. In contrast, GBCA treatment alone and simultaneous 7-T MRI exposure showed no evidence of enhanced $\gamma \mathrm{H} 2 \mathrm{AX}$ levels in peripheral lymphocytes. While Fiechter et al. [13] reported a significant increase in $\gamma \mathrm{H} 2 \mathrm{AX}$ after gadobutrol-enhanced MRI exposure, our current results support the findings of Brandt et al. [16] and Reddig et al. [19], who reported no induction of $\gamma \mathrm{H} 2 \mathrm{AX}$ foci in patients 5-30 min after contrast-enhanced MRI.

As Gd- and GBCA-induced cytotoxicity has been described for various cell types [5, 26, 30], we determined the level of cell death induced by different structural classes of GBCAs and investigated a potentially enhancing effect in combination with 7-T MRI. The apoptosis rate of lymphocytes was analysed $24 \mathrm{~h}$ after MRI exposure while GBCAs were still present. At a dose of $2 \mathrm{mM}$, a significant increase in cell death was only observed for the investigated linear, non-ionic gadodiamide, while a concentration of $20 \mathrm{mM}$ induced apoptosis for all three linear GBCAs. This cytotoxic effect occurred independently of MRI exposure and was detectable to a stronger extent by the level of DNA synthesis determined after $72 \mathrm{~h}$ in activated lymphocytes. At a dose of $20 \mathrm{mM}$, analysed classes of linear GBCAs led to a strong reduction in the proliferation rate, whereas no decrease was observed for the two macrocyclic GBCAs included in our study. However, additional markers need to be assessed to clarify this observation in activated lymphocytes.

A surprising finding was a slight decrease in apoptosis when lymphocytes were cultured in the presence of the macrocyclic gadobutrol (Fig. 2b). This decrease seems difficult to explain, as no mechanism for potential MRI-induced primary or secondary genotoxic or cytotoxic effects is known. To investigate this effect more deeply in further studies, it could be helpful to perform additional apoptosis assays, such as caspase 3 activity, which was not part of this study.

Furthermore, the exposure conditions required for cytotoxicity assessment in our in vitro experiments differed in comparision to GBCA pharmacokinetics in vivo. Upon injection in vivo GBCA rapidly distributes to the extracellular space and is eliminated from the blood with a half-life of approximately $1.5 \mathrm{~h}$ in subjects with normal renal function [31, 32]. Therefore, the drawing of reliable conclusions from our in vitro data for clinical routines is difficult.

As shown by Cho et al. [30], exposure of human lymphocytes to non-chelated $\mathrm{GdCl}_{3}$ led to a dose-dependent induction of apoptosis and DNA damage, which were further enhanced when samples were co-exposed to $\mathrm{Gd}$ and an extremely low-frequency electromagnetic field. Our cytotoxicity data compare well with previously described dissociation characteristics of toxic free $\mathrm{Gd}^{3+}$ from chemically different GBCA chelates. In general, macrocyclic GBCAs are described as being more stable than linear compounds [5, 33]. Analysing the $\mathrm{Gd}^{3+}$ release of multiple GBCAs in human serum over a period of 15 days, Frenzel et al. [34] observed an increase of free $\mathrm{Gd}^{3+}$ from ionic and especially non-ionic linear GBCAs, whereas all macrocyclic GBCAs remained stable. A correlation between osmolality of GBCA and cytotoxicity, as described for porcine renal epithelial cells by Heinrich et al. [26], was not observed in our study. Our results confirmed increased toxicity induced by the group of investigated linear GBCAs compared to the two analysed macrocyclic ones. Nevertheless, further in vitro and in vivo studies are needed to evaluate all commercially available MRI contrast agents and to detect possible mechanisms of toxicity or potential interaction in combination with high- and ultra-high-field MRI.

Limitations of this study include the circumstances that only a limited amount of analyses could be conducted within this experimental setup, and no conclusions regarding different cell types, different DNA lesions or long-term effects in vivo can be drawn. Furthermore, effects due to ingredients other than the pure substance of the GBCA cannot be fully excluded. Since no exact mechanisms about potential MRI-induced primary or secondary genotoxic or cytotoxic effects are known, it is difficult to choose the most sensitive assay and best point in time. Until now, experimental procedures are mainly motivated by studies investigating ionising radiation. The exposure conditions required for cytotoxicity assessment in our in vitro experiments differed regarding 
GBCA pharmacokinetics in vivo, as mentioned above. Therefore, the genotoxic and cytotoxic impact of different GBCAs in MRI needs to be further examined, in particular regarding $\mathrm{Gd}$ tissue retention and possible pathological effects, especially under more reliable in vivo conditions.

In conclusion, our in vitro data demonstrate no induction of $\gamma \mathrm{H} 2 \mathrm{AX}$ foci in isolated human lymphocytes after contrast-enhanced 7-T MRI exposure with respect to the five investigated GBCAs. Enhanced cytotoxicity was observed for the three tested linear GBCAs compared to the two macrocyclic ones, but no further amplified cell damage was determined when GBCA exposure was combined with 7-T MRI.

\section{Additional file}

Additional file 1: Table S1. Raw data for Fig. 1a, $\mathrm{yH} 2 \mathrm{AX}$ level after native and contrast-enhanced CT exposure in vitro. Table S2. Raw data for Fig. 1b, apoptosis rate after native and contrast-enhanced CT exposure in vitro. Table S3. Raw data for Fig. 1c, proliferation rate after native and contrast-enhanced CT exposure in vitro. Table S4. Raw data for Fig. 2a, $\mathrm{YH} 2 \mathrm{AX}$ level after native and contrast-enhanced 7-T magnetic resonance (MR) exposure in vitro. Table S5. Raw data for Fig. 2b, apoptosis rate after native and contrast-enhanced 7-T MR exposure in vitro. Table S6. Raw data for Fig. 2c, proliferation rate after native and contrast-enhanced 7-T MR exposure in vitro. (DOCX $63 \mathrm{~kb}$ )

\section{Abbreviations}

${ }^{3}[\mathrm{H}]-\mathrm{TdR}$ : Tritiated thymidine; CPT: Camptothecin; CT: Computed tomography; DSB: Double-strand break; GBCA: Gadolinium-based contrast agent; ICA: lodinated contrast agent; MRI: Magnetic resonance imaging; PBS: Phosphate-buffered saline; PHA: Phytohemagglutinin; PI: Propidium iodide; $Y H 2 A X$ : Phosphorylated histone H2AX

\section{Availability of data and materials}

The datasets supporting the conclusions of this article are included within the article and its Additional file 1: Tables S1-S6.

\section{Funding}

This work has not received any funding.

\begin{abstract}
Authors' contributions
Conceptualisation: BF, OS, DR, AR. Formal analysis: BF, MF, DR, AR. Investigation: BF, FG, MF, AR. Methodology: BF, OS, DR, AR. Project administration: BF, FG, MF, AR. Resources: OS, DR, DR. Supervision: AR. Validation: BF, AR. Visualisation: BF, AR. Writing, original draft: BF, AR. Writing, review and editing: all authors. All authors read and approved the final manuscript.
\end{abstract}

\section{Ethics approval and consent to participate}

This in vitro study was approved by the local ethics committee and written informed consent was obtained from all participants.

\section{Consent for publication}

Not applicable.

\section{Competing interests}

D. Roggenbuck is a shareholder of Medipan $\mathrm{GmbH}$ and has a patent PCT/ EP2012/06499, US 8,835,122, AU11168799.2 issued. O.S. reports grants from European Commission during the course of the study and grants from Siemens Healthcare. This support, however, is not related to the subject of the current study. The remaining authors declare no conflict of interest.

\section{Publisher's Note}

Springer Nature remains neutral with regard to jurisdictional claims in published maps and institutional affiliations.

\section{Author details}

${ }^{1}$ Department of Radiology and Nuclear Medicine, Otto von Guericke University Magdeburg, 39120 Magdeburg, Germany. ${ }^{2}$ Department of Biomedical Magnetic Resonance, Otto von Guericke University Magdeburg, 39120 Magdeburg, Germany. ${ }^{3}$ Leibniz Institute for Neurobiology, 39118 Magdeburg, Germany. ${ }^{4}$ Center for Behavioral Brain Sciences, Otto von Guericke University Magdeburg, 39118 Magdeburg, Germany. ${ }^{5}$ German Center for Neurodegenerative Disease, Site Magdeburg, 39120 Magdeburg, Germany. ${ }^{6}$ Medipan GmbH, 15827 Dahlewitz, Berlin, Germany. ${ }^{7}$ Institute of Biotechnology, Brandenburg University of Technology Cottbus-Senftenberg, 01958 Senftenberg, Germany. ${ }^{8}$ Institute of Molecular and Clinical Immunology, Otto von Guericke University Magdeburg, Leipziger Str. 44, 39120 Magdeburg, Germany.

Received: 17 May 2018 Accepted: 9 October 2018

Published online: 28 November 2018

\section{References}

1. OECD (2014) 46. Magnetic resonance imaging (MRI) exams, total per 1000 population. https://doi.org/10.1787/mri-exam-total-table-2014-1-en

2. Grand View Research Inc. (2017) Magnetic resonance imaging (MRI) market analysis by architecture (open and closed), by field strength (low, medium, high), by application (brain \& neurological, spine \& musculoskeletal, vascular, abdominal \& pelvic, cardiac, breast), and segment forecasts. http://www.grandviewresearch.com/industry-analysis/magneticresonance-imaging-market

3. Wang Y, Alkasab TK, Narin O et al (2011) Incidence of nephrogenic systemic fibrosis after adoption of restrictive gadolinium-based contrast agent guidelines. Radiology 260:105-111

4. Kanda T, Ishii K, Kawaguchi H, Kitajima K, Takenaka D (2014) High signal intensity in the dentate nucleus and globus pallidus on unenhanced T1weighted MR images: relationship with increasing cumulative dose of a gadolinium-based contrast material. Radiology 270:834-841

5. Rogosnitzky M, Branch S (2016) Gadolinium-based contrast agent toxicity: a review of known and proposed mechanisms. BioMetals 29:365-76

6. Radbruch A, Haase R, Kieslich PJ et al (2017) No signal intensity increase in the dentate nucleus on unenhanced T1-weighted MR images after more than 20 serial injections of macrocyclic gadolinium-based contrast agents. Radiology 282:699-707

7. Vijayalaxmi, Fatahi M, Speck O (2015) Magnetic resonance imaging (MRI): A review of genetic damage investigations. Mutat Res Rev Mutat Res 764:51-63

8. Hill MA, O'Neill P, McKenna WG (2016) Comments on potential health effects of MRI-induced DNA lesions: quality is more important to consider than quantity. Eur Heart J Cardiovasc Imaging 17:1230-1238

9. Foster KR, Moulder JE, Budinger TF (2017) Will an MRI examination damage your genes? Radiat Res 187:1-6

10. Fatahi M, Reddig A, Friebe B, Reinhold D, Speck O (2017) MRI and genetic damage: an update. Curr Radiol Rep 5:20

11. Simi S, Ballardin M, Casella M et al (2008) Is the genotoxic effect of magnetic resonance negligible? Low persistence of micronucleus frequency in lymphocytes of individuals after cardiac scan. Mutat Res 645:39-43

12. Lee JW, Kim MS, Kim YJ, Choi YJ, Lee Y, Chung HW (2011) Genotoxic effects of $3 \mathrm{~T}$ magnetic resonance imaging in cultured human lymphocytes. Bioelectromagnetics 32:535-542

13. Fiechter M, Stehli J, Fuchs TA, Dougoud S, Gaemperli O, Kaufmann PA (2013) Impact of cardiac magnetic resonance imaging on human lymphocyte DNA integrity. Eur Heart J 34:2340-2345

14. Lancellotti P, Nchimi A, Delierneux C et al (2015) Biological effects of cardiac magnetic resonance on human blood cells. Circ Cardiovasc Imaging 8:e003697

15. Szerencsi Á, Kubinyi G, Váliczkó É et al (2013) DNA integrity of human leukocytes after magnetic resonance imaging. Int J Radiat Biol 89:870-876

16. Brand M, Ellmann S, Sommer M et al (2015) Influence of cardiac MR imaging on DNA double-strand breaks in human blood lymphocytes. Radiology 277:406-412 
17. Reddig A, Fatahi M, Friebe B et al (2015) Analysis of DNA double-strand breaks and cytotoxicity after 7 tesla magnetic resonance imaging of isolated human lymphocytes. PLoS One 10:e0132702

18. Fatahi M, Reddig A, Vijayalaxmi et al (2016) DNA double-strand breaks and micronuclei in human blood lymphocytes after repeated whole body exposures to 7T magnetic resonance imaging. Neuroimage 133:288-293

19. Reddig A, Fatahi M, Roggenbuck D et al (2017) Impact of in vivo highfield-strength and ultra-high-field-strength MR imaging on DNA doublestrand-break formation in human lymphocytes. Radiology 282:782-789

20. Grudzenski S, Kuefner MA, Heckmann MB, Uder M, Löbrich M (2009) Contrast medium-enhanced radiation damage caused by CT examinations. Radiology 253:706-714

21. Pathe C, Eble K, Schmitz-Beuting D et al (2011) The presence of iodinated contrast agents amplifies DNA radiation damage in computed tomography. Contrast Media Mol Imaging 6:507-513

22. Piechowiak El, Peter JF, Kleb B, Klose KJ, Heverhagen JT (2015) Intravenous iodinated contrast agents amplify DNA radiation damage at $C$. Radiology 275:692-697

23. Jost G, Golfier S, Pietsch $\mathrm{H}$ et al (2009) The influence of $x$-ray contrast agents in computed tomography on the induction of dicentrics and gamma-H2AX foci in lymphocytes of human blood samples. Phys Med Biol 54:6029-6039

24. Yildiz S, Cece H, Kaya I et al (2011) Impact of contrast enhanced MRI on lymphocyte DNA damage and serum visfatin level. Clin Biochem 44:975-979

25. Witzleb E (1987) Functions of the vascular system. In: Schmidt RF, Thews G (eds) Human Physiology, 23rd edn. Springer Berlin Heidelberg, Berlin, Heidelberg, $p 490$

26. Heinrich MC, Kuhlmann MK, Kohlbacher S et al (2007) Cytotoxicity of iodinated and gadolinium-based contrast agents in renal tubular cells at angiographic concentrations: in vitro study. Radiology 242:425-434

27. Willitzki A, Lorenz S, Hiemann R et al (2013) Fully automated analysis of chemically induced $\mathrm{YH} 2 \mathrm{AX}$ foci in human peripheral blood mononuclear cells by indirect immunofluorescence. Cytometry A 83:1017-1026

28. Schwenzer NF, Bantleon R, Maurer B et al (2007) Detection of DNA doublestrand breaks using gammaH2AX after MRI exposure at 3 tesla: an in vitro study. J Magn Reson Imaging 26:1308-1314

29. Erdamar H, Gürgel A, Namuslu M et al (2014) Is magnetic resonance imaging really innocent? Clin Chem Lab Med 52:24114916

30. Cho S, Lee Y, Lee S, Choi YJ, Chung HW (2014) Enhanced cytotoxic and genotoxic effects of gadolinium following ELF-EMF irradiation in human lymphocytes. Drug Chem Toxicol 37:440-447

31. Aime S, Caravan P (2009) Biodistribution of gadolinium-based contrast agents, including gadolinium deposition. J Magn Reson Imaging 30:1259-1267

32. Caschera L, Lazzara A, Piergallini L, Ricci D, Tuscano B, Vanzulli A (2016) Contrast agents in diagnostic imaging: Present and future. Pharmacol Res 110:65-75

33. Fraum TJ, Ludwig DR, Bashir MR, Fowler KJ (2017) Gadolinium-based contrast agents: a comprehensive risk assessment. J Magn Reson Imaging 46:338-353

34. Frenzel T, Lengsfeld P, Schirmer H, Hütter J, Weinmann HJ (2008) Stability of gadolinium-based magnetic resonance imaging contrast agents in human serum at 37 degrees C. Invest Radiol 43:817-828

\section{Submit your manuscript to a SpringerOpen ${ }^{\circ}$ journal and benefit from:}

- Convenient online submission

- Rigorous peer review

- Open access: articles freely available online

- High visibility within the field

- Retaining the copyright to your article

Submit your next manuscript at $\boldsymbol{\nabla}$ springeropen.com 